\title{
Phase behavior of mixtures of oppositely charged protein nanoparticles at asymmetric charge ratios
}

\author{
P. Maarten Biesheuvel, ${ }^{1,2}$ Saskia Lindhoud, ${ }^{1}$ Martien A. Cohen Stuart, ${ }^{1}$ and Renko de Vries ${ }^{1}$ \\ ${ }^{1}$ Laboratory of Physical Chemistry and Colloid Science, Wageningen University, Dreijenplein 6, 6703 HB Wageningen, The Netherlands \\ ${ }^{2}$ Max Planck Institute for Colloids and Surfaces, Am Mühlenberg 1, 14476 Potsdam, Germany
}

(Received 20 January 2006; published 25 April 2006)

\begin{abstract}
We present experimental and theoretical results for the phase behavior of mixtures of oppositely charged globular protein molecules in aqueous solutions containing monovalent salt. These colloidal mixtures are interesting model systems, on the one hand for electrolyte solutions ("colloidal ionic liquids"), and on the other for mixtures of oppositely charged (bio)macromolecules, colloids, micelles, etc., with the range of the electrostatic interactions (Debye length) easily tunable from much smaller to much larger than the particle size, simply by adding different amounts of monovalent salt. In this paper we investigate the phase behavior of such mixtures in the case that equally sized colloids have a large difference in charge magnitude. This is possible at any mixing ratio because small ions compensate any colloidal charge asymmetry. Our experimental system is based on lysozyme, a positively charged "hard" globular protein molecule, and succinylated lysozyme, a chemical modification of lysozyme which is negatively charged. By changing the solution $p \mathrm{H}$ we can adjust the ratio of charge between the two molecules. To describe phase separation into a dilute phase and a dense "complex" phase, a thermodynamic model is set up in which we combine the Carnahan-Starling-van der Waals equation of state with a heterogeneous Poisson-Boltzmann cell model and include the possibility that protein molecules adjust their charge when they move from one phase to the other (charge regulation). The theory uses the nonelectrostatic attraction strength as the only adjustable parameter and reasonably well reproduces the data in that complexation is only possible at intermediate $p \mathrm{H}$, not too asymmetric mixing ratios, and low enough ionic strength and temperature.
\end{abstract}

DOI: 10.1103/PhysRevE.73.041408

PACS number(s): 82.70.Dd, 82.35.Rs, 87.14.Ee

\section{INTRODUCTION}

Complexation of oppositely charged macroions, such as micelles, polyelectrolytes, and/or colloids is observed in a large range of biological systems, and is exploited in many technologies such as microencapsulation by complex coacervates, soluble DNA-polycation complexes for nonviral gene transfer, and thin film fabrication by layer-by-layer deposition of oppositely charged polyelectrolytes.

Because of the combination of a repulsion between likecharged particles and an attraction between oppositely charged particles, these systems show very interesting phase behavior ("associative phase separation") where both soluble as well as macroscopic "complex" phases can be observed when the attractive electrostatic forces are strong enough. Compared to systems involving charged polymer chains, relatively little attention has been paid to systems involving oppositely charged spherical particles, such as proteins and colloids. Nevertheless, such systems are interesting both as model systems, and because of possible technological and scientific implications [1-3]. For example, Leunissen et al. [4] recently showed how to exploit electrostatic complexation to assemble various novel colloidal cocrystals with particles differing in both charge and size. Furthermore, the cytosol of living cells may be viewed as a mixture of proteins of different charge (sign). Recently it was suggested that, at least for prokaryotes, the cytoplasm is quite close to the phase boundary for macroscopic phase separation [5]. Complexation of the minority of basic proteins with acidic globular proteins may contribute significantly to the stability or instability of the cytoplasm.
To study phase behavior in such mixtures, simple experimental model systems are required. Ideally these are based on spherical particles, homogeneously charged, and with the range and strength of the interaction forces easily tunable. For equilibrium structures to be formed, the range of the attraction should be of the order of the particle radius or larger. To that end, Leunissen et al. [4] and Bartlett and Campbell [6] who use polymethyl methacrylate PMMA particles of a radius of $\sim 1 \mu \mathrm{m}$, work in highly deionized solvents of a Debye length $\kappa^{-1}$ of $\sim 0.2$ to $1.6 \mu \mathrm{m}$. Raşa et al. [7] use particles that are of the order of $10^{2}$ smaller (size $\sim 15 \mathrm{~nm}$ ), and by working in ethanol realize a Debye length $\kappa^{-1}$ of 10-20 nm. Our approach [8] is to go to even smaller "colloidal ions" by using globular (approximately spherical) protein molecules, of a radius of $\sim 2 \mathrm{~nm}$. To obtain Debye lengths in the relevant range we can simply work in aqueous solution, and can realize values for $0.2<\kappa R<2$ by adding monovalent salt at reasonable values for the ionic strength $(1-100 \mathrm{mM})$. By changing $p \mathrm{H}$ we can quite easily tune the charge on the colloids, which will be the topic of this report.

In a previous report [8] we introduced our model system which is based on lysozyme, a "hard" globular protein molecule, positively charged for $p \mathrm{H}$ below its isoelectric point $(p I \sim 11)$, and succinylated lysozyme, a chemical modification of lysozyme which is negatively charged above $p \mathrm{H}$ $\sim 4.5$. Because the negative molecule is synthesized from the positive one (by chemically modifying some of the amino acid residues), we believe that to a good approximation the molecules are the same, except for their charge. In Ref. [8] we studied the macroscopic phase behavior of this system at 
a $p \mathrm{H}$ where the two molecules are about equally charged (in magnitude). In the present paper we will investigate the behavior of this model system at asymmetric charge ratios by bringing the $p \mathrm{H}$ toward the isoelectric point of one of the protein molecules.

To describe the experimental observations (either incipient conditions for phase separation, or amount of complexed material), we use a thermodynamic theory developed in Ref. [8] for mixtures of charged spherical colloids that interact across an aqueous solution containing co- and counterions. Other theoretical approaches are described in Ref. [8] while molecular dynamics simulations of mixtures of oppositely charged spheres were made by Liu and Luijten [9] and Caballero et al. [10] In the model of Ref. [8] the charge on the colloids was calculated under the condition of infinite dilution, and assumed constant, independent of the presence of nearby colloids. At $p \mathrm{H} \mathrm{7-8}$ this is a very good approximation for the two types of lysozyme molecules, as their charge is (in that $p \mathrm{H}$ range) quite independent of local proton concentration and therefore also independent of the proximity to other molecules. However, this is not generally true, certainly not near the isoelectric point where the charge of a protein molecule depends strongly on the local proton concentration. In the present paper we include the possibility that nearby colloids influence the proton concentration near a protein molecule and thus its charge ("charge regulation"). In the thermodynamic functions we must additionally include a chemical term (a contribution that contains the proton adsorption energy, and the entropy of the two states of the amino acid, charged and uncharged). We will compare theoretical results based on the assumption that the protein molecules have the same charge in both phases [constant-charge (CC) model], with the situation that they adjust their charge according to local conditions [i.e., proton concentration; the charge-regulation (CR) model].

In this paper we use for the protein molecules alternatively the terms "particle" and "colloid." The dense phase is also called the "complex" phase; the term "supernatant" is sometimes used for the dilute phase. In the following sections we describe the thermodynamic theory, the experimental program and results, and the comparison of theory with the data.

\section{THEORY}

A theoretical model is described for phase separation in mixtures of oppositely charged protein nanoparticles in aqueous solution. The model is an extension of that described in Ref. [8], by including the ionizable character of the protein molecules in more detail, namely, by considering that the protein charge not only is a function of ionic strength and $p \mathrm{H}$, but also of the density and composition of the respective phase (dilute phase or complex phase), i.e., the proximity to other protein molecules ( $\mathrm{CR}$ model), because the proton concentration near the protein molecule is modified, and via the proton adsorption reactions the charge is affected. The fixedcharge (or CC) model calculates the protein charge based on a dilute protein solution, that is, when the diffuse layer around the molecule extends undisturbed to infinity, and the protein charge is influenced only by $p \mathrm{H}$ and ionic strength.

Typically, within the complex phase the electrostatic potential at the surface of the protein molecule is closer to zero than in the solution phase; thus, in the CR model both molecules have a higher charge (in magnitude). Therefore, compared to a fixed-charge approach, allowing for charge regulation leads to an increased range in which complexation is thermodynamically possible. Around $p \mathrm{H} 7.5$ (as in Ref. [8]) both molecules have a charge that is rather independent of $p \mathrm{H}$, and assuming a fixed protein charge is a good approximation. However, at $p \mathrm{H}$ closer to the isoelectric point a protein molecule becomes more titratable (charge more dependent on local proton concentration), and charge regulation may become important.

\section{Heterogeneous cell model}

To describe the electrostatic interactions, we use the heterogeneous Poisson-Boltzmann (PB) cell model of Ref. [11], where it was applied to charge-regulating coil-like polyelectrolytes using a cylindrical geometry without linearizing the PB equation. In Ref. [8] the model was applied to oppositely charged spheres of a fixed charge in the Debye-Hückel (DH) limit. Here, we use the DH limit as well, while we will analyze both the $\mathrm{CC}$ and $\mathrm{CR}$ models.

In the heterogeneous cell model we consider an envelope of solvent plus small ions around each charged colloid; the envelope plus colloid together constitute one spherical cell. For a bidisperse mixture we consider two types of cells. The $\mathrm{PB}$ equation is solved in a spherical geometry within the solvent envelope between colloid and cell edge. The boundary conditions are Gauss' law at the surface of the colloid, a common value for the electrostatic potential $y$ at the edge of all cells, and finally overall electroneutrality of all cells (of the two types) combined, which translates into a relation between the two (different) field strengths at the edge of the (two) different types of cells (and the number concentration of each type of cell). The heterogeneous cell model simplifies to the traditional one-component cell model if only one type of colloid is present, and, for very dilute conditions, results in the classical expression for the potential around a single charged spherical colloid. Compared to a onecomponent cell model, the field strength at the edge of the cell, however, is no longer set to zero a priori, but is selfconsistently obtained from the requirement of overall electroneutrality. Because the magnitude of the electrostatic potential is a measure of the electrostatic repulsion between the colloids, and (the square of) the field strength is a measure of the electrostatic attraction, the protein molecules can electrostatically both attract and repel. This is different from onecomponent cell models where we always have electrostatic repulsion between the colloids. The cell model has the advantage that it is not necessary to describe the positions of the different colloids relative to one another, which is required when the model is based on binary, or two-body, interaction potentials (such as the Yukawa potential). In the cell model interactions of a given colloid with all other colloids are included via the boundary condition at the edge of the cell. 
Because the protein molecules are of equal radius $a$, it is quite natural to assume in the cell model that the two types of cells have the same outer radius $b$ after which the volume density of all colloids combined, $\phi$, is given by

$$
\phi=\frac{a^{3}}{b^{3}} .
$$

We use the Debye-Hückel approximation of linearizing the $\mathrm{PB}$ equation to be solved in the spherical, annular, space between the protein molecule surface (at $r=a$ ) and the edge of the cell $(r=b)$, which results for the electrostatic potential $y_{p, i}$ at the surface of the colloid, where $r=a$, in cell $i$, in

$$
y_{p, i}=\frac{\kappa \lambda_{B}}{t_{h}+\kappa a}\left(\frac{t_{h}}{\kappa a} Z_{i}+\left(1-t_{h}^{2}\right) \frac{Q_{1}}{\phi} \sum_{i=1,2} Z_{i} \phi_{i}\right)
$$

where

$$
Q_{1}=\left[1-\frac{a}{b}-\left(\frac{1}{\kappa b}-\kappa a\right) t_{h}\right]^{-1}, \quad t_{h}=\tanh (\kappa b-\kappa a) .
$$

Here $\kappa$ is the inverse of the Debye length, given by $\kappa^{2}$ $=8 \pi \lambda_{B} n_{\infty}$, when all ions are monovalent. The ionic strength $n_{\infty}\left(=c_{\infty} N_{\text {av }}\right.$ with $c_{\infty}$ in $\left.\mathrm{mM}\right)$ is defined in a virtual colloid-free phase containing only the small ions, in equilibrium with the (two) phase(s) containing the colloids (thus, we fix the ion chemical potential as $\left.\ln n_{\infty}\right) . N_{\text {av }}$ is Avogadro's number, and $\lambda_{B}$ the Bjerrum length (in water $\lambda_{B}=0.72 \mathrm{~nm}$ ).

The protein charge $Z_{i}$ is obtained according to the Tanford titration model, which considers a spherical particle with a smeared-out surface charge $[8,12-15]$. The total protein charge $Z_{i}$ is given by a summation over the six types of ionizable amino acid groups of number $q_{i}$, charge sign $z_{i}$, and ionization degree $\alpha_{i}$,

$$
Z=\sum_{i} q_{i} z_{i} \alpha_{i} .
$$

The ionization degree $\alpha_{i}$ of each amino acid residue relates to the electrostatic surface potential $y_{p}$ according to

$$
\alpha_{i}=\frac{1}{1+10^{z_{i}\left(p \mathrm{H}-p \mathrm{~K}_{i}\right)} e^{z_{i} y_{p}}}
$$

where $p \mathrm{H}$ is the background $p \mathrm{H}$ (a measure of the chemical potential of protons in the system) and $p \mathrm{~K}$ the intrinsic $p \mathrm{~K}$ value, a thermodynamic number directly related to the (nonelectrostatic) adsorption energy of a proton to a certain amino acid group. Now, whereas in the CC model we calculate the charge on the basis of assuming an infinitely dilute solution, in the CR model we self-consistently relate $y_{p}$ to $Z$ using the heterogeneous cell model. Typically, this implies that because the potentials $y_{p}$ are closer to zero in the dense phase than in solution, the charge of both the negative and the positive colloids is higher in magnitude. By using Eqs. (4) and (5) we include the influence of (the charge of) all the amino acids on the ionization degree of each titratable group via the smeared-out surface potential $y_{p}$. Via $y_{p}$ also the dependence of ionic strength on $\alpha_{i}$ is included. However, local variations in potential over the protein surface, and a pertur- bation in $p \mathrm{~K}$ due to a (nonelectrostatic) influence of nearby amino acid residues is not considered in the model [16].

\section{Electrostatic contribution to the thermodynamic functions}

Solving the cell model analytically results in an expression for the electrostatic contribution to the osmotic pressure of the protein mixture, $\Pi$, given by $[8]$

$$
\Pi_{\mathrm{el}}=\frac{\kappa^{2} b \lambda_{B}}{6 v} \frac{1-t_{h}^{2}}{\left(t_{h}+\kappa a\right)^{2}}\left[\frac{Q_{1} Q_{2}}{\phi}\left(\sum_{i=1,2} \phi_{i} Z_{i}\right)^{2}-\sum_{i=1,2} \phi_{i} Z_{i}^{2}\right]
$$

with

$$
\begin{aligned}
Q_{2}= & 2 t_{h}\left(t_{h}+\kappa a\right)+1-t_{h}^{2}+Q_{1}\left(t_{h}+\kappa a\right) \\
& \times\left[\frac{a}{\kappa b^{2}}+\frac{1}{\kappa^{2} b^{2}} t_{h}-\left(\frac{1}{\kappa b}-\kappa a\right)\left(1-t_{h}^{2}\right)\right],
\end{aligned}
$$

irrespective of whether the colloids are charge regulating or not. The electric contribution to the potential, $\mu_{\mathrm{el}}$, remains unmodified as well [8]:

$$
\mu_{i, \mathrm{el}}=\frac{1}{2} Z_{i} y_{i}+\frac{1}{2}\left(Z_{i}-Z_{j}\right) \frac{Q_{1} \kappa \lambda_{B} \phi_{j}}{\phi^{2}} \frac{1-t_{h}^{2}}{t_{h}+\kappa a} \sum_{i=1,2} \phi_{i} Z_{i}+\Pi_{\mathrm{el}} \frac{v}{\phi}
$$

but for the charge-regulation model we must add to $\mu$ a chemical contribution because of the varying ionization degrees, given by $[13,14,17,18]$

$$
\mu_{i, \text { chem }}=-Z_{i} y_{i}+\sum_{j} q_{j} \ln \left(1-\alpha_{j}\right)
$$

with the summation running over the six types of ionizable amino acid residues.

\section{Nonelectrostatic contribution to the thermodynamic functions}

The nonelectrostatic contributions are described using the Carnahan-Starling-van der Waals equation of state which gives as contribution to the osmotic pressure

$$
\Pi_{\text {nonel }} v=\phi \frac{1+\phi+\phi^{2}-\phi^{3}}{(1-\phi)^{3}}-\chi \phi^{2},
$$

and to the chemical potential

$$
\mu_{i, \text { nonel }}=\ln \phi_{i}+\frac{\phi\left(8-9 \phi+3 \phi^{2}\right)}{(1-\phi)^{3}}-2 \chi \phi .
$$

In the above equations, $\phi$ is the overall volume fraction of protein molecules and $\phi_{i}$ is the volume fraction of each individual component. A nonelectrostatic, temperaturedependent, attraction between the colloids is included via the parameter $\chi$. Note that the attraction term and the volume exclusion term contain only the total protein concentration $\phi$ : we do not distinguish between the two types of molecules at this level. To describe phase separation in (onecomponent) protein solutions, the use of Eqs. (10) and (11) 
was already proposed by Taratuta et al. [19] and Petsev et al. [20] We extend that approach to multicomponent protein solutions, and explicitly describe the electrostatic interactions (not, e.g., via a modification of the $\chi$ parameters).

At equilibrium, the osmotic pressure $\Pi$ (combining electrostatic and nonelectrostatic contributions) is equal in both phases, as well as the potential $\mu$ for the two components. Finally, overall mass balances for the two protein molecules are required, such as Eq. (25) in Ref. [8].

\section{Fixed number of small ions}

In most of the calculations we will assume a fixed background ionic strength $c_{\infty}$, given by the amount of added salt. However, it turns out that in the experiments shown in Fig. 4 below this assumption fails below $\sim 2 \mathrm{mM}$ salt. Instead, we must consider that the number of ions in the system is fixed. As all experiments at low ionic strength were done at low $p \mathrm{H}$ values it was $\mathrm{HCl}$ that was used to adjust $p \mathrm{H}$; therefore it is the number of cations $(\mathrm{Na})$ that is fixed (i.e., not influenced by adjusting $p \mathrm{H})$ and will be considered in the ion balances.

In the low-potential, Debye-Hückel, approach, the protein charge $Z$ (let us assume it to be positive) is 50\% compensated by a cation deficiency, and $50 \%$ by an excess of anions near the colloid (and vice versa for a negatively charged colloid). Thus, the total cation balance reads

$$
n_{C, \text { added }}=n_{\infty}-\frac{1}{2 v} \sum_{j} \zeta_{j} \sum_{i} \phi_{i, j} Z_{i, j}
$$

where $n$ is the cation concentration, $n_{\infty}$ the ionic strength used in the definition of the Debye length $\kappa^{-1}$, and $\zeta$ the relative volume of phase $j(0<\zeta<1)$. In one calculation we will assume that $q^{*}$ cations are codissolved with each protein molecule; in that case we must add to the left-hand side of Eq. (12) the term $+\phi_{0} q^{*} / v$, where $\phi_{0}$ is the total protein volume fraction.

\section{Summary of input parameters required in the theory}

To summarize, the theoretical model requires the following input parameters. First, for each protein type we need the number of each of the six types of ionizable groups, $q_{i}$, and the intrinsic $p \mathrm{~K}$ value of each of them. Next, we need to assume a certain volume per molecule, $v$, and a certain value for the attraction parameter $\chi$ (which depends on temperature). Then we can make a calculation as a function of the overall volume fraction of each protein in the system $\left(\phi_{i, 0}\right.$, which gives us the overall mixing ratio $\left.\alpha_{0}\right), p \mathrm{H}$, and Debye length $\kappa^{-1}$, the latter either directly based on the added salt concentration $c_{\text {salt }}\left(n_{\infty}\right)$, or indirectly via Eq. (12). The theory then predicts whether or not phase separation occurs, and if so, predicts the composition and density of each of the two phases. All parameters in the theory can be obtained from other sources: $q_{i}$ follows directly from the amino acid composition of each protein, while $p \mathrm{~K}$ values [21] and the protein volume [22] are obtained from the literature. We use the attraction parameter $\chi$ as a freely adjustable parameter

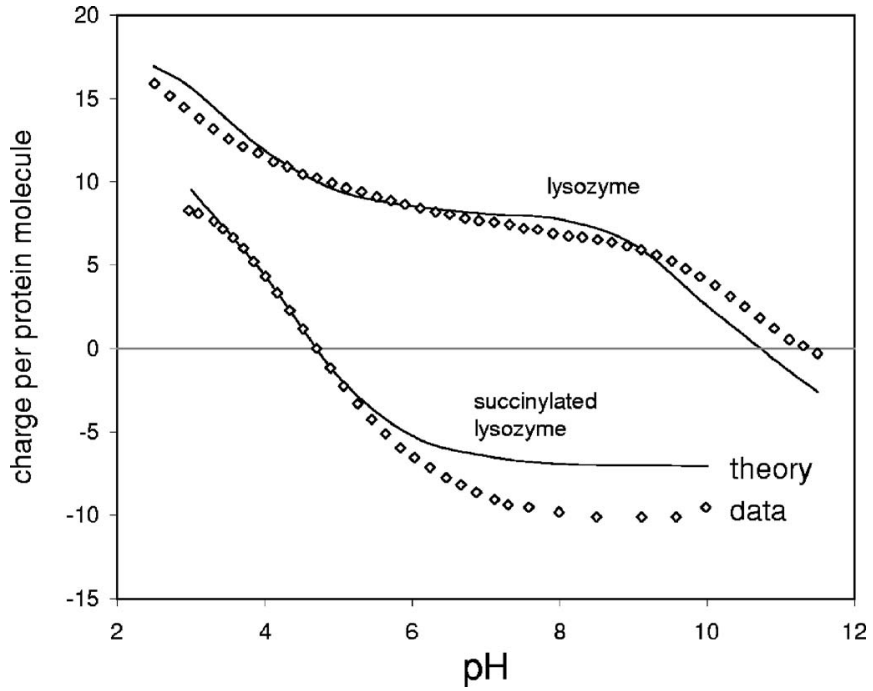

FIG. 1. Protein charge as function of $p \mathrm{H}$ for (succinylated) lysozyme $\left(c_{\infty}=50 \mathrm{mM}\right)[15,23]$.

though the range of values we use is comparable to those found for pure-lysozyme systems, as discussed in Ref. [8].

\section{EXPERIMENT}

\section{Proteins}

Succinylated lysozyme (SL) with molecular weight $\left(\mathrm{MW}_{\mathrm{SL}}\right) 15.2 \mathrm{kDa}$ is synthesized from hen egg white lysozyme $\left(\mathrm{L} ; \mathrm{MW}_{\mathrm{L}}=14.3 \mathrm{kDa}\right)$ by a procedure described in Ref. [23]. Subsequently it is dialyzed three times against a large excess of distilled water. For both molecules we use in the calculations a volume of $v=37 \mathrm{~nm}^{3}$, as in Ref. [8]. The overall mixing ratio $\alpha_{0}$ is the overall number concentration of $\mathrm{L}$ divided by the total protein number concentration, and, because the volume per molecule is assumed the same for $\mathrm{L}$ and $\mathrm{SL}$, it is also equal to the volume fraction of $\mathrm{L}$ over the total volume fraction of $\mathrm{L}+\mathrm{SL}$ [note that $\alpha$ is the ionization degree; see Eq. (5)]. We use the same titration model for $\mathrm{L}$ and SL as in Refs. [8] and [15] but note that in Ref. [8] the charge was calculated for a molecule in an infinitely dilute solution, using $t_{h}=1$ in Eq. (2), which in the present work will be done for calculations with the CC model only. In the $\mathrm{CR}$ model the charge per molecule is different in the dilute and complex phases.

Equations (4) and (5) require the intrinsic $p \mathrm{~K}$ value of each of the amino acids, $p \mathrm{~K}_{i}$, and the number of each of these amino acids per protein molecule, $q_{i}$. For the anionic amino acids we use $p \mathrm{~K}_{D}=p \mathrm{~K}_{E}=4.4$ and $p \mathrm{~K}_{Y}=10$; for the cationic amino acids $p \mathrm{~K}_{R}=12, p \mathrm{~K}_{H}=6.5$ and $p \mathrm{~K}_{K}=10$ [21]. For lysozyme we have $q_{D}=7, q_{E}=2, q_{Y}=3, q_{R}=11, q_{H}=1$, $q_{K}=6$; for SL $q_{D}=16, q_{E}=2, q_{Y}=0, q_{R}=11, q_{H}=1$, and $q_{K}$ $=0$. These values result in theoretical isoelectric points of $p \mathrm{I}_{L}=10.7$ and $p \mathrm{I}_{\mathrm{SL}}=4.7$, respectively. For illustration purposes, we show in Fig. 1 the measured and calculated protein charge as a function of $p \mathrm{H}$, for an ionic strength $c_{\infty}$ $=50 \mathrm{mM}$, taken from Refs. [15] and [23].

\section{Phase separation}

The protein molecules are dissolved in separate (onecomponent) stock solutions of a certain ionic strength 


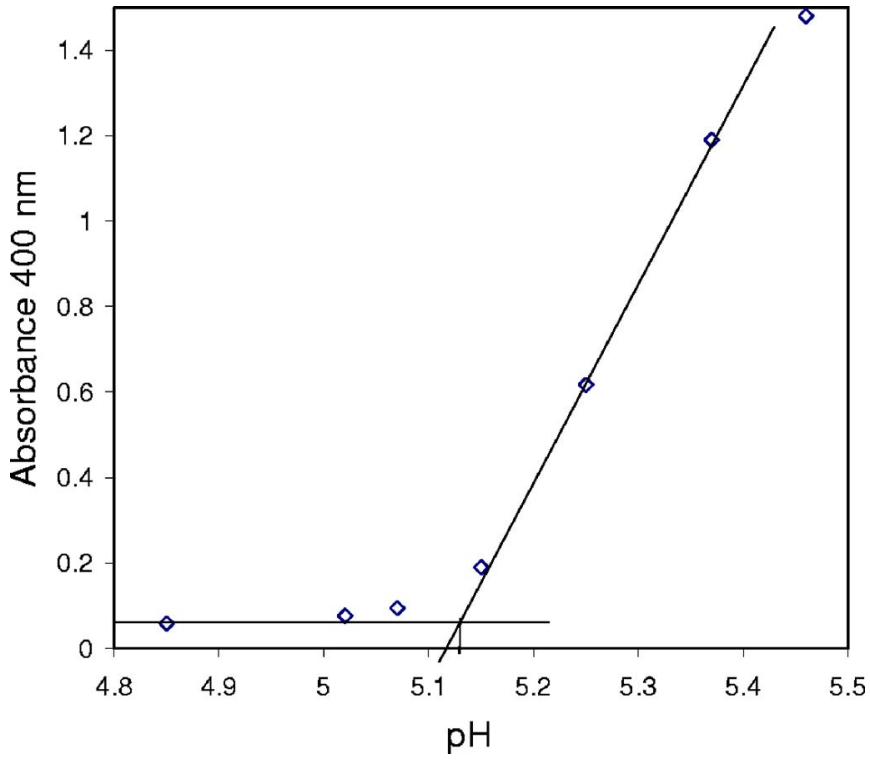

FIG. 2. (Color online) Typical example of method I to determine phase boundary from absorbance measurements $\left(\alpha_{0}=0.5, \phi_{0}\right.$ $=0.2 \mathrm{vol} \%$, no added salt, room temperature).

$(\mathrm{NaCl})$, after which the solutions are mixed in certain proportions in a $25 \mathrm{ml}$ stirred glass tube. We assume that the dialyzed and freeze-dried polymer is free of water and contaminants and will use the molar masses of the protein as given above to calculate the protein concentration and volume fraction (using $v=37 \mathrm{~nm}^{3}$ per molecule). After each $p \mathrm{H}$ adjustment (with either $\mathrm{HCl}$ or $\mathrm{NaOH}$ ) we wait for several minutes before taking a measurement. The following three methods were used to study phase separation in the mixed protein solutions:

METHOD I. Spectrophotometrically, the absorbance is measured at $400 \mathrm{~nm}$ (turbidity) as function of $p \mathrm{H}$. To obtain the critical condition for the onset of phase separation a range of measurements is made at different $p \mathrm{H}$ values and the critical value $p \mathrm{H}_{\text {crit }}$ obtained from the construction illustrated in Fig. 2. This procedure is used for the data in Figs. 3 and 4 below.

METHOD II. Mixtures are transferred into capped plastic Eppendorf tubes of $1.5 \mathrm{ml}$, centrifuged for $30 \mathrm{~min}$ at 10000 rotations/min, and left to equilibrate for $1 \mathrm{~h}$, after which the protein concentration in the supernatant phase was determined spectrophotometrically $(281.5 \mathrm{~nm})$ using a calibration curve.

METHOD III. Mixtures are prepared, transferred into capped glass tubes $(\sim 3 \mathrm{ml})$, and placed in a stirred water bath. The temperature of the bath is increased slowly, $\sim 0.2{ }^{\circ} \mathrm{C} / \mathrm{min}$, and we determine by eye at which temperature the sample becomes completely transparent, thus obtaining the clarification temperature $T_{\text {clarify }}[8,19,24]$. Results of this method are plotted in Fig. 6 below.

\section{RESULTS AND DISCUSSION}

\section{Method I: Turbidity experiments}

In a first set of experiments we analyze phase behavior as a function of $p \mathrm{H}$ and composition, at a given protein concen-

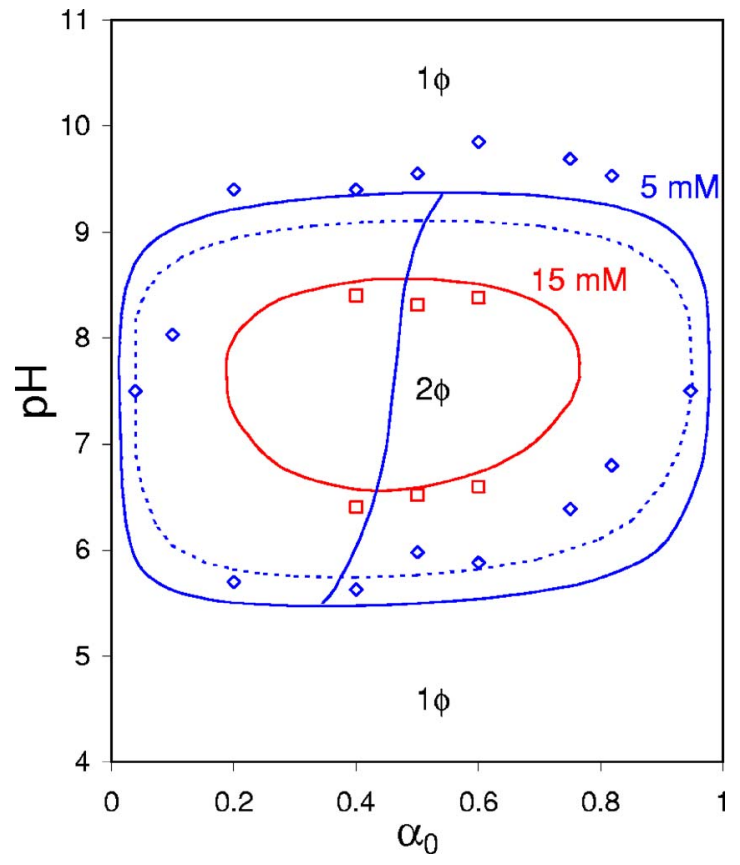

FIG. 3. (Color online) $p \mathrm{H}$-composition phase diagram for lysozyme-succinylated lysozyme at $0.1 \mathrm{vol} \%$ total protein concentration at two values of the ionic strength (points denote onset of phase separation according to method I; see Fig. 2). Lines are based on the CR model (solid, $\chi=14$; dashed, $\chi=13$ and $5 \mathrm{mM}$ ). The almost vertical line gives for $5 \mathrm{mM}$ salt and $\chi=14$ for each $p \mathrm{H}$ the mixing ratio $\alpha_{0}$ at which the number of complexed molecules is at a maximum. $1 \phi$ denotes the one-phase, non-phase-separated, region; phase separation is observed in the $2 \phi$ region.

tration $\left(\phi_{0}=0.1 \mathrm{vol} \%\right)$ and ionic strength $(5$ and $15 \mathrm{mM})$; see Fig. 3. A closed phase diagram is expected in the $\alpha_{0}-p \mathrm{H}$ plane because we can always move out of the twophase " $2 \phi$ " region by increasing or decreasing $p \mathrm{H}$ or mixing ratio sufficiently. This is the case because at very low or very high $p \mathrm{H}$ all molecules have the same charge sign; and at increasingly asymmetric mixing ratios the system approaches a one-component system, which is for our conditions always stable (single-protein systems will phase separate when temperatures are very low and/or ionic strength or protein concentration is very high $[19,30])$. Indeed, a closed $2 \phi$ region is experimentally observed; see Fig. 3 .

In addition, we expected the closed region to be tilted upward, with the $p \mathrm{H}$ window for phase separation shifted downward at a low mixing ratio $\alpha_{0}$, and upward at high $\alpha_{0}$, because, for instance, with decreasing $p \mathrm{H}$ the lysozyme molecules increase their charge and the SL molecules decrease their charge (in magnitude), and for charge neutrality in the complex we would then need more SL than L molecules, which suggests that the optimum mixing ratio shifts to lower amounts of $\mathrm{L}$; thus the range of values of the mixing ratio $\alpha_{0}$ for which phase separation occurs would decrease. However, though there certainly is an upward tilt in the experimental data, it is not very pronounced and for both low and high $p \mathrm{H}$, we find a wide $\alpha_{0}$ window around $\alpha_{0}=0.5$ where the $p \mathrm{H}$ for the onset of phase separation is rather independent of $\alpha_{0}$. The theoretical curves (based on the CR model) show an even slighter tilt. 

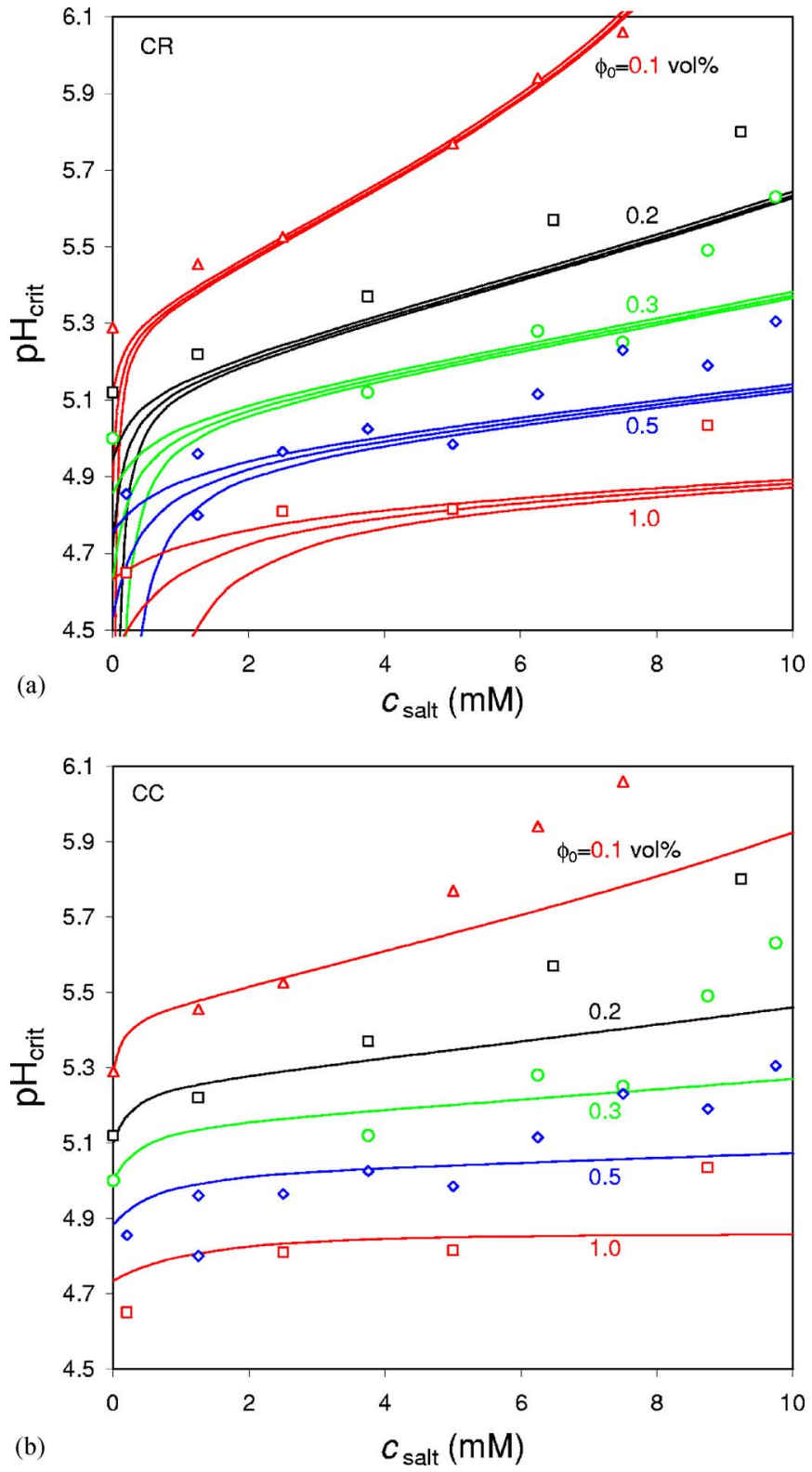

FIG. 4. (Color online) $p \mathrm{H}$-added salt concentration phase diagram for lysozyme-succinylated lysozyme at $0.1 \mathrm{vol} \%$ total protein concentration at a symmetric mixing ratio, $\alpha_{0}=0.5$ (method I). Above each theoretical curve, or set of data points, the system is phase separated (a) CR model and (b) CC model. In (a) each set of three theoretical curves is based from bottom to top on fixed background ionic strength, fixed number of cations, and the same with an additional $q^{*}=2$ cations per protein molecule.

Now, because here, both theoretically and experimentally, only the onset of phase separation is reported, it might be interesting to investigate the conditions for maximum phase separation, and see if an upward tilt is perhaps observed in that parameter: if we increase $p \mathrm{H}$, will the mixing ratio $\alpha_{0}$ at which the complexed amount is at a maximum, $p \mathrm{H}_{\text {maxcompl }}$, steadily shift upward? The approximately vertical curve of $\alpha_{0, \text { maxcompl }}-p \mathrm{H}$ in Fig. 3 gives the result, and shows that although $\alpha_{0 \text {,maxcompl }}$ shifts upward with increasing $p \mathrm{H}$, the effect is not very strong. Clearly, the intuitive idea that with increasing $p \mathrm{H}$ the optimum condition for complexation shifts to higher relative concentrations of $\mathrm{L}, \alpha_{0}$, is only slightly borne out by the experiments and theory.

The solid lines in Fig. 3 are based on the CR model and $\chi=14$ for a best fit to the data $[\chi=14$ is also the derived value in Fig. 6(b) for the CR model at room temperature, $T \sim 22{ }^{\circ} \mathrm{C}$ ]. We also plot our prediction for $\chi=13$ (dashed curve) as this is the best-fit value in the experiments shown later in Fig. 4. Though this value gives a reasonable prediction at $5 \mathrm{mM}$, at $15 \mathrm{mM}$ absence of phase separation is predicted, in disagreement with the experimental observation. Calculations in Fig. 3 are all based on the CR model; the CC model predicts a somewhat less extended phase boundary in the vertical $p \mathrm{H}$ direction (not shown), and therefore describes the data somewhat less accurately.

In a next set of experiments the aim was to find a phaseseparated system at a $p \mathrm{H}$ value as far away as possible from the optimum range around $p \mathrm{H} \mathrm{7.5.} \mathrm{We} \mathrm{chose} \mathrm{to} \mathrm{lower} p \mathrm{H}$ toward the isoelectric point of succinylated lysozyme because this requires less addition of $\mathrm{HCl}$ than the required amount of $\mathrm{NaOH}$ addition in the case of going to the $p \mathrm{I}$ of lysozyme, $\sim 11.5$. Experiments were done at a symmetric mixing ratio $\alpha_{0}=0.5$, because the analysis in Fig. 3 suggests that lowering $\alpha_{0}$ does not expand the region of phase separation to lower $p \mathrm{H}$ very significantly. Second, we also decided to lower the ionic strength because a low ionic strength favors phase separation. Experimental results are shown in Fig. 4 together with three sets of calculations. In Fig. 4(a) we include charge regulation, optimize the $\chi$ value to $\chi=13$, and use three different ways to include the cations, namely, as follows. For each protein concentration $\phi_{0}$, the lowest curve is based on a calculation in which it is assumed that the ionic strength $n_{\infty}$ is given by the added amount of salt. Though the data are rather well described down to $\sim 2 \mathrm{mM}$ salt, we find a clear deviation at lower ionic strengths. The theory predicts that upon lowering the ionic strength we will for all $p \mathrm{H}$ values ultimately end up in a phase-separated regime. The data, however, suggest that even without adding salt still a distinct critical $p \mathrm{H}$ value is found below which the system is a single phase (no complex phase formed). Therefore we include in the calculation the fact that the number of cations is finite, by using Eq. (12), which results in the middle curves in Fig. 4(a). This adjustment already gives a significantly improved result, with at $0 \mathrm{mM}$ added salt finite values for $p \mathrm{H}_{\text {crit }}$ predicted, in line with the data. However, the predicted values for $p \mathrm{H}_{\text {crit }}$ are still too low. A final modification to the theory is to include the possibility that with each protein molecule $q^{*}=2$ cations are codissolved. Interestingly, this modification brings the theoretical prediction rather close to the experimental data [see each of the upper curves in Fig. 4(a)] which suggests that, despite dialysis, a small number of ions is retained in the protein sample. This is not unlikely as also during dialysis the protein molecules tend to remain charged, which requires counterions to remain nearby. Only ion exchange, or dialysis against an extremely ion-free dialyzing fluid, will result in an essentially ion-free protein sample.

Of the data in Fig. 4, one data point is at a $p \mathrm{H}$ value around the isoelectric point of succinylated lysozyme (namely, at the highest protein concentration of $1 \mathrm{vol} \%$, at 
$0.2 \mathrm{mM}$ salt), which was the lowest $p \mathrm{H}_{\text {crit }}$ we could obtain. At higher protein concentrations we did not obtain a suitable turbidity data set such as shown in Fig. 2, but instead upon lowering $p \mathrm{H}$ to values below $p \mathrm{H} 4.5$ we found that the turbidity increased rapidly again. After that, the sample remained turbid at all $p \mathrm{H}$ values (not reversible). It seems that at these low $p \mathrm{H}$ values and high protein concentrations there is a nonreversible attraction, not accounted for in the current theoretical approach, perhaps related to some protein unfolding. Therefore we will leave these conditions out of the present analysis.

A second use of the data set of Fig. 4 is to compare the predictive power of the CR model, Fig. 4(a), with that of the CC model, Fig. 4(b). To that end, we fitted in panel Fig. 4(b) the CC model to the data, making use of the same assumptions with respect to the small ions as in the best fit for panel $a$ (namely, taking account of the finite number of cations, and with $q^{*}=2$ ), and separately fit the attraction strength $\chi$ $\left(\chi_{\mathrm{CC}}=14.5\right)$. We find that the CR model gives a somewhat better prediction of the phase boundary than the CC model, the most notable difference being that the theoretical curves in Fig. 4(b) (CC model) are almost horizontal, in contrast to both the experiments and to the theoretical curves in Fig. 4(a) (CR model) where the model predicts a positive slope which rather closely matches the experiment. This comparison of CC and CR models suggests that indeed the molecules do adjust their charge to the local proton concentration when moving from the complex phase to the supernatant and vice versa.

\section{Method II: Centrifugation experiments}

In a next set of experiments, again at room temperature, we do not measure the phase boundary (onset of phase separation) but instead the total amount of molecules in the complex phase, or alternatively, see Fig. 5, the percentage of molecules remaining in solution after centrifuging off the complex phase, $\phi_{s} / \phi_{0}$ (where $\phi_{s}$ is the supernatant concentration, and $\phi_{0}$ the overall concentration). To convert the data (for absorbance at $281.5 \mathrm{~nm}$ ) to $\phi_{s} / \phi_{0}$, we rescale each data set such that at low $p \mathrm{H} \phi_{s} / \phi_{0}$ equals $100 \%$. Figure 5 presents data and theory for $\phi_{s} / \phi_{0}$ as a function of $p \mathrm{H}$ and ionic strength. Data and theory both show that at extreme enough $p \mathrm{H}$ we have a stable system (single phase, $100 \%$ in solution) whereas a dip in $\phi_{s} / \phi_{0}$ develops at intermediate $p \mathrm{H}$. The dip expands to a wider $p \mathrm{H}$ range and increases in depth with decreasing ionic strength, in both theory and the experiments. With a fitted value of $\chi=13$ the theory describes the three datasets best, and also predicts the minimum value of the supernatant concentration for each ionic strength reasonably well. However, there are significant deviations too: the change of $\phi_{s} / \phi_{0}$ with ionic strength is overestimated, and the extent of complexation at higher $p \mathrm{H}$ (beyond $p \mathrm{H} \sim 8.5$ ) is significantly overestimated. This latter effect might well be due to the fact that the titration model, which is rather accurate for $4<p \mathrm{H}<6$ for both molecules (see Fig. 1), starts to deviate from the data at $p \mathrm{H}>8$. A better titration model [16] might improve the quality of the model in this $p \mathrm{H}$ range. Using a slightly lower $\chi$ (namely, $\chi=12.5$ ) significantly im-

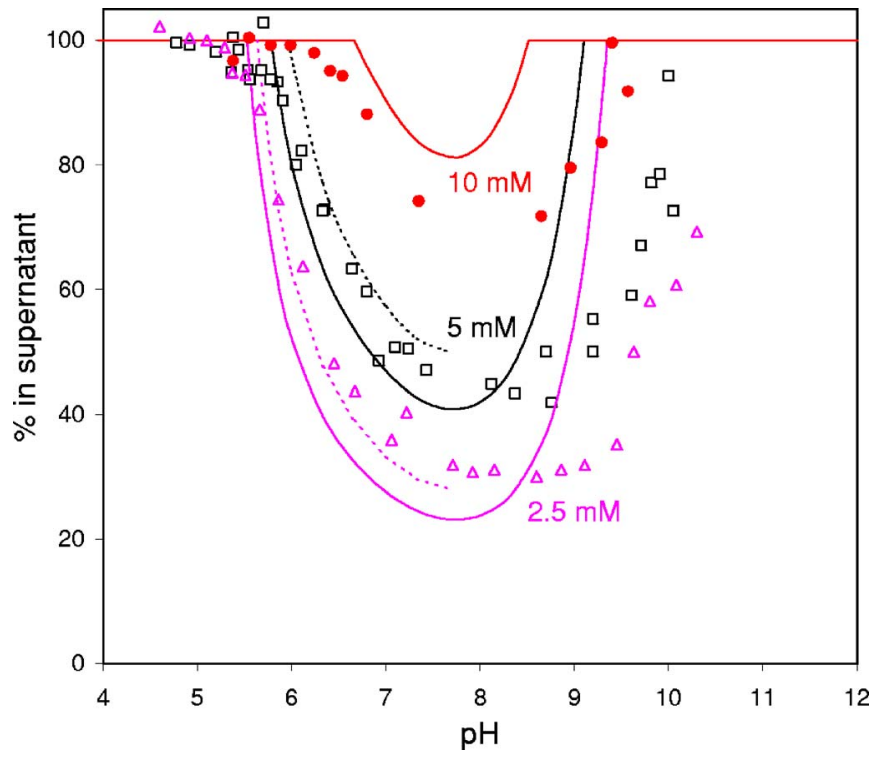

FIG. 5. (Color online) Percentage of total protein remaining in supernatant phase as function of $p \mathrm{H}$ and ionic strength $\left(\phi_{0}\right.$ $=0.1 \mathrm{vol} \%, \alpha_{0}=0.5$, method II). Theoretical curves based on the CR model with $\chi=13$ (dashed curves $\chi=12.5$ ).

proves the fit for 2.5 and $5 \mathrm{mM}$ salt for $p \mathrm{H}<8$ (dashed lines in Fig. 5) but would, over the entire range of $p \mathrm{H}$ and ionic strength, not be an overall improvement.

\section{Method III: Temperature influence}

Finally, we determined the clarification temperature of a phase-separated, turbid sample by increasing the temperature slowly, similar to the approach in Refs. $[8,19,24]$, for a variety of values of $p \mathrm{H}$ and total protein concentration (mixing ratio $\alpha_{0}=0.5$; ionic strength $=5 \mathrm{mM}$ ). Subsequently, we used the same procedure as in Ref. [8], namely, to plot theoretical $\chi$ values for the onset of phase separation at each experimental condition $(p \mathrm{H}$, ionic strength, etc.) against the experimental temperature. If the theory is valid, a single $\chi(T)$ curve should describe the data; if not, the various points will be scattered across space. We performed this procedure to see, first of all, if the theory is able to describe the experiment also for asymmetric charge ratios, and second, if we can distinguish between the $\mathrm{CC}$ model and the $\mathrm{CR}$ model in their predictive power. Results are presented in Fig. 6 . We see that with both models a well-defined $\chi(T)$ correlation is found, with about the same extent of scatter around the best-fit curve. In both cases, the scatter in the data is much larger below $15^{\circ} \mathrm{C}$ than at higher temperatures. Indeed, at low temperature it was more difficult to ascertain experimentally whether or not the sample had become completely transparent; at higher temperatures the transition from turbid to transparent was more distinct (i.e., occurred over a smaller temperature interval).

The predictive power of both models can best be com-

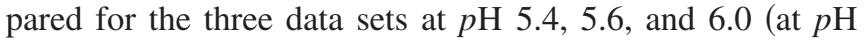
5.0, 5.2, and 5.8 the models cannot be distinguished in their predictive power). Making this analysis we see that the CR model (full curves) gives a somewhat better prediction than 

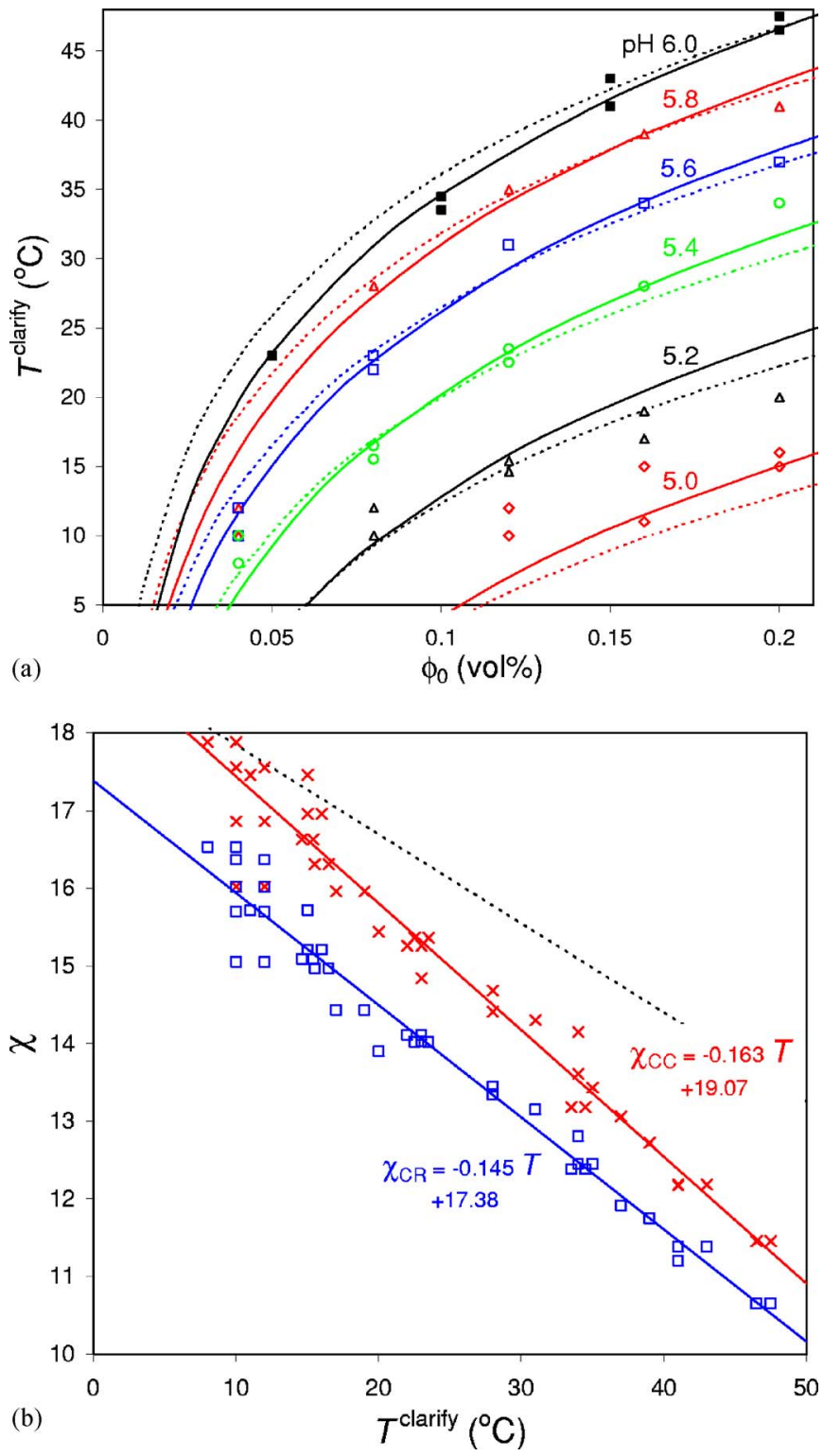

FIG. 6. (Color online) (a) Clarification temperature as function of $p \mathrm{H}$ and total protein concentration, $\phi_{0}\left(\alpha_{0}=0.5 ; c_{\text {salt }}=5 \mathrm{mM}\right)$. Solid lines show calculation results for the charge regulation, CR, model and dashed lines for a fixed protein charge (CC model). (b) $\chi(T)$ correlation for the $\mathrm{CC}$ (crosses) and CR models (squares). Also shown (dashed line) is the $\chi(T)$ correlation from Ref. [8].

the CC model (dashed lines). Thus, very tentatively, also this set of experiments suggests that the protein molecules do indeed charge regulate, and adjust their charge when moving into and out of the dense phase.

\section{Discussion of nonelectrostatic attractive force}

Though the theory reasonably well describes the data of protein phase separation, at least two problems remain. First of all, the fact that different numerical values for $\chi$ have to be used throughout this work, as well as in Ref. [8], and secondly that the origin (of the temperature dependence) of the attractive force is not very well known.
On the first point, it must be remarked that when we jointly consider the various data sets in this paper together with those in Ref. [8], it is not possible to use a single value for $\chi$, but different values need to be used for the different experimental protocols (even when using the same method). For instance, the best-fit values for $\chi$ are different in Figs. 3 and 4 , while there is also a difference in the $\chi(T)$-curve [see Fig. 6(b)], between the experiments in this paper $(5 \mathrm{mM}$ salt, $p \mathrm{H} \mathrm{5.0-6.0)}$ and those in Ref. [8] (5-40 mM, $p \mathrm{H}$ 7.5). This shows that the theoretical model is certainly still insufficient in describing the underlying forces in this system accurately.

To improve the model, and possibly to describe the temperature dependence to some extent, one could consider the following aspects. One cause for the influence of temperature on the $2 \phi$ stability is possibly a decrease in protein charge with increasing temperature via an influence on the $p \mathrm{~K}$ values. However, Schaller and Robertson [25] conclude from their study on turkey ovomucoid third domain (a protein) that "no significant changes in $p \mathrm{~K}_{a}$ were observed over the $25{ }^{\circ} \mathrm{C}$ temperature change employed... which leads us to conclude that none of the apparent ionization enthalpies $\Delta H_{\text {ion }}$ exceeds $2 \mathrm{kcal} / \mathrm{mol}$." For sperm whale metmyoglobin, Breslow and Gurd [26] find $\Delta H_{\text {ion }}$ ranging from $2.5 \mathrm{kcal} / \mathrm{mol}$ for the carboxylic groups to $10.7 \mathrm{kcal} / \mathrm{mol}$ for the amine groups. Using $\partial p \mathrm{~K} / d T=-\Delta H_{\text {ion }} /(\ln 10) \mathrm{RT}^{2}$ (with $\Delta H_{\text {ion }}$ in $\mathrm{J} / \mathrm{mol}$ and $R$ the gas constant) $[26,27]$ and using $T$ $=300 \mathrm{~K}$, we calculate that these values for $\Delta H_{\text {ion }}$ result in a $p \mathrm{~K}$ decrease [13] of 0.15 to 0.65 over a $25^{\circ} \mathrm{C}$ temperature range (similar data for $\Delta H_{\text {ion }}$ are in Ref. [28]). Indeed these values are not very large and will not readily result in the observed significant effect of temperature on complexation behavior. Another effect of temperature on the electrostatic interaction is via the permittivity $\epsilon$ of water. However, $\epsilon T$, which is the relevant parameter that shows up in the Debye length, only varies very weakly with temperature [29] $(3 \%$ over $25^{\circ} \mathrm{C}$; thus $\kappa$ only changes by $1.5 \%$ ). A final suggestion for an electrostatic origin of the attraction and its temperature dependence is that due to the inhomogeneous charge distribution over the surface of the protein molecules a dipolar attraction develops with a positive patch on one molecule orienting toward a negative patch on the next. At higher temperature, with increasing thermal energy, the tendency of the protein molecules to diffuse and rotate increases (a larger force would be necessary to keep them locked in, without rotating), and therefore the dipolar attraction might be significantly reduced. At this point we have no means to quantify the strength of a possible dipolar attraction.

However, most probably the attractive term, and its temperature dependence, "which can hardly be reconciled with a purely electrostatic approach" [30] have a chemical origin related to nonelectrostatic (solvation, hydrophobic) interactions. Chemical modification of proteins is a known method to increase hydrophobicity, which is, e.g., used to compare native and modified proteins in adsorption experiments $[31,32]$. Our succinylated lysozyme is most likely more hydrophobic than (native) lysozyme, resulting in increased hydrophobic interactions. Possibly also a nonelectrostatic effect related to (solvation of) the small ions can contribute to this attractive term [33]. We have attempted in the present work to separate out the mean-field electrostatic forces and volume 
and entropy effects from this attractive protein-protein interaction "[of which] the molecular origins are poorly understood" [33] and to empirically quantify its strength and temperature dependence.

\section{CONCLUSIONS}

Mixtures of lysozyme and succinylated lysozyme phase separate under suitable conditions: low ionic strength, low temperature, symmetric mixing ratio, high protein concentration, and $p \mathrm{H}$ near to conditions when they both have high, opposite, charge, thus ideally around $p \mathrm{H}$ 7.5. Moving away from optimal conditions, fewer and fewer molecules remain in the complex phase, and ultimately phase separation ceases. A thermodynamical model based on a heterogeneous cell model for the electrostatic interactions describes the experimental data well, also at asymmetric charge ratios.

A theoretical model that includes charge regulation gives a somewhat better fit to the data than in case a fixed charge is assumed, which suggests that the protonation reactions that determine the charge of a protein molecule are sufficiently fast compared to the rate of protein exchange between supernatant and complex phase. For ionic strengths below $2 \mathrm{mM}$ and at protein concentrations of $0.1 \mathrm{vol} \%(\sim 1 \mathrm{~g} / \mathrm{l})$ a constant background ionic strength (given by the amount of added salt) can no longer be assumed, but instead we must consider that the number of cations and anions in the system is fixed, as well as that the dialyzed and dried protein samples contain a small number of cations $(\sim 2$ per protein molecule). A temperature-dependent nonelectrostatic attractive term is required to bring the model into quantitative agreement with the data.

\section{ACKNOWLEDGMENTS}

This research was financially supported by NWO, Netherlands Organization for Scientific Research, and the Alexander von Humboldt Foundation, Germany.
[1] A. van Blaaderen, Nature (London) 439, 545 (2006).

[2] D. Frenkel, Nat. Mater. 5, 85 (2006).

[3] W. Lin, M. Kobayashi, M. Skarba, C. Mu, P. Galletto, and M. Borkovec, Langmuir 22, 1038 (2006).

[4] M. E. Leunissen, C. G. Christova, A. P. Hynninen, C. P. Royall, A. I. Campbell, A. Imhof, M. Dijkstra, R. van Roij, and A. van Blaaderen, Nature (London) 437, 235 (2005).

[5] F. N. Braun, S. Paulsen, R. P. Sear, and P. B. Warren, Phys. Rev. Lett. 94, 178105 (2005).

[6] P. Bartlett and A. I. Campbell, Phys. Rev. Lett. 95, 128302 (2005).

[7] M. Raşa, A. P. Philipse, and J. D. Meeldijk, J. Colloid Interface Sci. 278, 115 (2004).

[8] P. M. Biesheuvel, S. Lindhoud, R. de Vries, and M. A. Cohen Stuart, Langmuir 22, 1291 (2006).

[9] J. Liu and E. Luijten, Phys. Rev. Lett. 93, 247802 (2004).

[10] J. B. Caballero, A. M. Puertas, A. Fernández-Barbero, and F. J. de las Nievas, J. Chem. Phys. 121, 2428 (2004).

[11] P. M. Biesheuvel and M. A. Cohen Stuart, Langmuir 20, 4764 (2004).

[12] D. Stigter and K. A. Dill, Biochemistry 29, 1262 (1999).

[13] D. Stigter, D. O. V. Alonso, and K. A. Dill, Proc. Natl. Acad. Sci. U.S.A. 88, 4176 (1991).

[14] D. O. V. Alonso, K. A. Dill, and D. Stigter, Biopolymers 31, 1631 (1991).

[15] P. M. Biesheuvel, M. van der Veen, and W. Norde, J. Phys. Chem. B 109, 4172 (2005).

[16] D. E. Kuehner, J. Engmann, F. Fergg, M. Wernick, H. W. Blanch, and J. M. Prausnitz, J. Phys. Chem. B 103, 1368
(1999)

[17] P. M. Biesheuvel, Eur. Phys. J. E 16, 353 (2005).

[18] P. M. Biesheuvel and A. Wittemann, J. Phys. Chem. B 109, 4209 (2005).

[19] V. G. Taratuta, A. Holschbach, G. M. Thurston, D. Blankschtein, and G. B. Benedek, J. Phys. Chem. 94, 2140 (1990).

[20] D. N. Petsev, X. Wu, O. Galkin, and P. G. Vekilov, J. Phys. Chem. B 107, 3921 (2003).

[21] L. Stryer, Biochemistry (Freeman, New York, 1995).

[22] F. L. González Flecha and V. Levi, Biochem. Mol. Biol. Educ. 31, 319 (2003).

[23] M. van der Veen, W. Norde, and M. A. Cohen Stuart, Colloids Surf. B 35, 33 (2004).

[24] C. Liu, N. Asherie, A. Lomakin, J. Pande, O. Ogun, and G. B. Benedek, Proc. Natl. Acad. Sci. U.S.A. 93, 377 (1996).

[25] W. Schaller and A. D. Robertson, Biochemistry 34, 4174 (1995).

[26] E. Breslow and F. R. N. Gurd, J. Biol. Chem. 237, 371 (1962).

[27] R. W. Ramette, J. Chem. Educ. 54, 280 (1977).

[28] M. Tanaka, H. Nomura, and F. Kawaizumi, Bull. Chem. Soc. Jpn. 65, 410 (1992).

[29] B. B. Owen, R. C. Miller, C. E. Milner, and H. L. Cogan, J. Phys. Chem. 65, 2065 (1961).

[30] R. Piazza, Curr. Opin. Colloid Interface Sci. 8, 515 (2004).

[31] S. Magdassi, A. Kamyshny, and A. Baszkin, J. Dispersion Sci. Technol. 22, 313 (2001).

[32] P. A. Wierenga, M. B. J. Meinders, M. R. Egmond, F. Voragen, and H. H. J. de Jongh, Langmuir 19, 8964 (2003).

[33] R. A. Curtis and L. Lue, Chem. Eng. Sci. 61, 907 (2006). 